\title{
Indoleamine 2, 3-dioxygenase regulation of immune response (Review)
}

\author{
HAO WU ${ }^{1}$, JIANPING GONG ${ }^{1}$ and YONG LIU ${ }^{2}$ \\ ${ }^{1}$ Chongqing Key Laboratory of Hepatobiliary Surgery and Department of Hepatobiliary Surgery, \\ Second Affiliated Hospital, Chongqing Medical University, Chongqing 400010; \\ ${ }^{2}$ Department of Hepatobiliary Surgery, The People's Hospital of Hechuan, Chongqing 401520, P.R. China
}

Received November 28, 2016; Accepted January 4, 2018

DOI: $10.3892 / \mathrm{mmr} .2018 .8537$

\begin{abstract}
Indoleamine 2, 3-dioxygenase (IDO) catalyzes the initial and rate-limiting step in the degradation pathway of the essential amino acid tryptophan and is expressed by professional antigen presenting cells (APCs), epithelial cells, vascular endothelium and tumor cells. IDO-mediated catabolic products, which are additionally termed 'kynurenines', exerts important immunosuppressive functions primarily via regulating $\mathrm{T}$ effector cell anergy and inducing the proliferation of $\mathrm{T}$ regulatory cells. This endogenous tolerogenic pathway has a critical effect on mediating the magnitude of immune responses under various stress conditions, including tumor, infection and transplantation. The present review evaluates the recent progress in elucidating how catabolism of tryptophan regulated by IDO modulates the immune response to inflammatory and immunological signals. Blocking this pathway may be a novel adjuvant therapeutic strategy for clinical application in immunotherapy.
\end{abstract}

Correspondence to: Dr Yong Liu, Department of Hepatobiliary Surgery, The People's Hospital of Hechuan, 1366 Xieran Avenue, Chongqing 401520, P.R. China

E-mail:wuhao_48@163.com

Abbreviations: IDO, Indoleamine 2, 3-dioxygenase; APCs, antigen presenting cells; STAT1, activated signal transducer and activator of transcription 1 ; NF- $\kappa \mathrm{B}$, nuclear factor-kappa B; iNOS, inducible nitric oxide synthase; mTOR, mammalian target of Rapamycin; GCN2, general control nondepressible-2; AhR, aryl hydrocarbon receptor; DCs, dendritic cells; eIF-2 $\alpha$, eukaryotic translation initiation factor $2 \alpha$; PKC- $\theta$, protein kinase C-sita; ILT3, Ig-like transcript 3; Tat, N-terminal domain of human immune deficiency virus-1 transactivator regulatory protein; JAKs, janus activated kinases; PI3K, phosphatidyl inositol 3-kinase; 1-MT, 1-methyl-DL-tryptophan; BIN-1, bridging integrator 1

Key words: indoleamine 2, 3-dioxygenase, immune regulation, regulatory $\mathrm{T}$ cell, tryptophan, kynurenine

\section{Contents}

1. Introduction

2. Properties of the IDO enzyme

3. IDO pathways and immune regulation

4. Role of IDO in immune suppression

5. Strategies to target IDO

6. Conclusions

\section{Introduction}

As a highly evolved biological response, immunoregulation not only coordinates inflammation and innate immunity, however may additionally modulate adaptive immunity and establish self-tolerance. Continuous access to nutrients is a primary requirement for cell proliferation, and controlling nutrient supply is an ancient survival strategy that may additionally regulate immune response. Indoleaine 2,3-dioxygenase (IDO) is a tryptophan enzyme composed of two-helical $\alpha$-domains with a heme group located between them (1-2). A total of two forms of the IDO gene (IDO1 and IDO2) have been identified, however, the majority of studies have investigated the function of IDO1, and the physiological role of IDO2 remains unclear.

The immune regulating properties of IDO were first described in the prevention of T-cell-mediated allogenetic fetus rejection in mice, verifying that IDO synthesized in placental cells protects the mammalian fetus from maternal T lymphocyte attack (3). The degradation of tryptophan and accumulation of tryptophan-derived catabolites by IDO may lead to the suppression of T-cell proliferation at mid-G1 phase, the inhibition of activated $\mathrm{T}$ effector cells, and the induction of T, B, and natural killer cell apoptosis $(1,3)$. IDO has previously been demonstrated to be expressed in various tissues, including human lung, placenta, and small intestine and is upregulated during inflammation. Physiologically, IDO is pivotal in regulating the immune response to antigenic challenges at mucosal surfaces in the digestive tract and lungs (4). Furthermore, the induction of IDO and the subsequent deprivation of tryptophan in the microenvironment, exerts an antiproliferative effect on T cells and infectious pathogens (3). IDO is recognized to be an authentic regulator of immunity in a variety of pathophysiological settings, including infections, 
transplantation and cancer $(1,5)$. The present review aimed to evaluate recent progress and evidence regarding how IDO activity impacts the immune responses to inflammatory and immunological signals.

\section{Properties of the IDO enzyme}

IDO, a 407-amino acid heme-containing cytoplasmic protein, is coded by the INDO gene located on chromosome $8 \mathrm{p} 12$ in humans. The enzyme is responsible for the primary mechanism of extra-hepatic tryptophan metabolism and is expressed in professional APCs, epithelial cells, vascular endothelium and tumor cells $(1,2,5)$. The IDO gene is regulated by upstream interferon (IFN)- $\gamma$ responsive elements that bind to activated signal transducer and activator of transcription 1 (STAT1), interferon regulatory factor-1 and nuclear factor- $\mathrm{\kappa B}$ $(\mathrm{NF}-\mathrm{\kappa B})(6)$. IDO activity is strongly associated with an environment enriched with redox active compounds, which are required to generate the active $\mathrm{Fe} 2^{+}$form for tryptophan metabolism. IDO enzymatically degrades tryptophan and other indoleamine compounds via oxidative cleavage of the pyrrole ring, resulting in an accumulation of downstream breakdown products of kynurenine, in addition to other defined metabolic products, which have been previously reported to exhibit biological activity in the immune system (Fig. 1) (7). Currently, the ratio of kynurenine/tryptophan is regarded as a method to determine the enzymatic activity of IDO. Furthermore, adding strong reductants (methylenum coeruleum, vitamin C) into the IDO culture system results in the maintenance of the superoxide anion, an accessory factor of IDO, at a high concentration, thus enhancing its activity. The enzymatic activity of IDO is mitigated by natural immunomodulators including nitric oxide, which are able to combine with the heme tetrapyrrole group (8).

IDO expression has been revealed to be markedly different between various cells depending on the specific cell type, the maturational state and the activation status of the cells. The catabolic pathway regulated by IDO activity results from activity of two different genes, termed IDO1 and IDO2. Mice and humans possess the two associated genes tightly in a syntenic region of chromosome 8 (9). The two genes exhibit sequence homology, however, they respond differently to various signals in distinct cell types, and their patterns of gene regulation and expression are not identical. Currently, IDO1 is the more comprehensively studied of the two genes, however IDO2 is gradually being recognized. It has previously been demonstrated that IDO expression and enzymatic activity is mediated by suppressor of cytokine signaling 3 (SOCS3), NF- $\mathrm{KB}$, DNAX-activation protein 12 and interferon regulatory factor- 8 pathways. In addition, IDO protein levels and activity are modulated posttranslationally via ubiquitination or protein nitration via inducible nitric oxide synthase (iNOS). Administration of iNOS blockers following transplantation has been demonstrated to improve allograft function and attenuate graft damage (10).

\section{IDO pathways and immune regulation}

Uncontrollable immune responses may be fatal, therefore the immune system is delicately balanced between immunity and tolerance. IDO, as a major inhibitor of the immune response, appears to be pivotal in imposing restrictions on potentially exaggerated inflammatory reactions to danger signals. IDO contributes to immune regulation via catalyzing tryptophan along the kynurenine pathway. IDO modifies the immune response via three pathways: i) by depleting tryptophan in the local microenvironment, IDO results in metabolic stress sensed by general control nondepressible-2 (GCN2) kinase and mammalian target of Rapamycin (mTOR), which eventually promotes anergy in responding $\mathrm{T}$ cells and directs the conversion of regulatory $\mathrm{T}$ cells (Treg) (11); ii) by producing tryptophan catabolism that binds to a natural ligand for the aryl hydrocarbon receptor (AhR), IDO similarly results in immunosuppressive effects on the immunogenicity of dendritic cell (DCs) reduction and the Treg conversion (12); iii) by inducing Treg function, IDO as a signaling protein shapes the immunological microenviroment in vivo. The following section identifies mechanisms by which IDO pathways are implicated in modulating immune regulation.

\section{IDO depletes tryptophan and produces bioactive downstream metabolites}

Effector pathways: GCN2 activation and mTOR suppression. IDO activity decreases the local concentration of tryptophan in the IDO-expressing cell and in the microenvironment surrounding nearby cells. Tryptophan depletion by IDO may act as a potential regulatory signal via two signaling pathways: activation of the molecular stress-response pathway, including GCN2 kinase, which directly binds to uncharged tryptophan tRNAs, and suppression of the mTOR kinase pathway, which is known to regulate immune reactions (13). DCs expressing IDO may induce the immunosuppressive activity of Tregs, however this effect is observed to be abrogated by genetic disruption of GCN2. Depletion of tryptophan has been demonstrated to mediate the activation of the GCN2 pathway for downregulating the $\mathrm{CD} 3 \zeta$-chain in $\mathrm{CD}^{+} \mathrm{T}$ cells and blocking Th17 cell differentiation (14). GCN2 blunts protein translation by phosphorylating its downstream target, initiating eukaryotic translation initiation factor $2 \alpha$ (eIF-2 $\alpha$ ), resulting in blockade readout of the majority of RNA transcripts, except for the LIP RNA transcript. IDO supports interleukin (IL)-6 production through GCN2 activation and was revealed to affect myeloid-derived suppressor cell function and tumor progression (15). A previous study in nephritis mouse models, conducted by Chaudhary et al (16), suggested that kidney injury of mice were improved by amino acid metabolism and protected from the autophagic response. The IFN- $\gamma$-mediated induction of IDO activity with subsequent activation of GCN2 is implied in the metabolic signaling process. These results outline the IDO-GCN2 pathway, as a critical negative feedback mechanism in glomerular stromal cells, via inducing autophagy that limits inflammatory renal pathological alterations. Therefore, the IDO-GCN2 pathway mediates amino-acid levels and may contribute to a generalized mechanism of regulating the immune response.

Amino acid withdrawal additionally has an effect on the nutrient-sensing mTOR pathway. IDO-mediated catabolism of tryptophan inhibits mTOR and $\mathrm{T}$ cell receptor protein kinase C-sita (PKC- $\theta$ ), which are regulatory targets of the master amino acid-sensing galactokinase 1 acting upstream of mTOR (17). Furthermore, in the tumor microenvironment, 


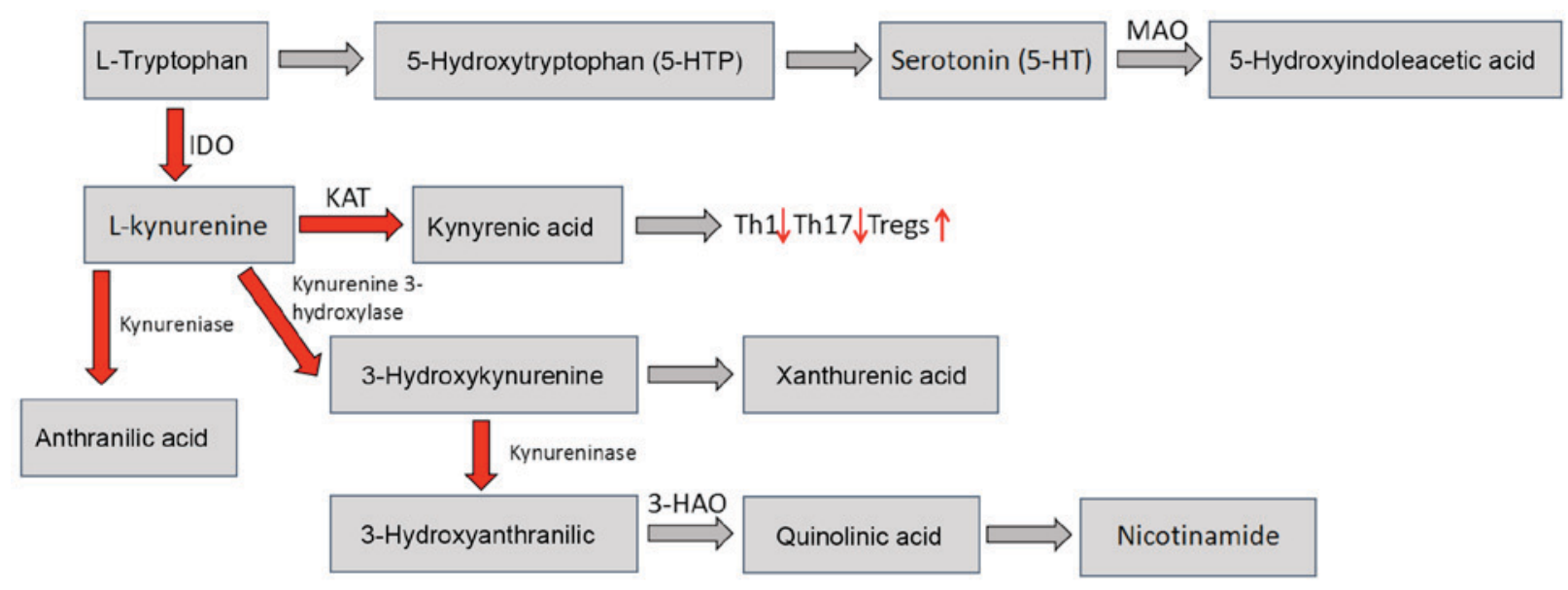

Figure 1. Metabolism of tryptophan. In vivo, $99 \%$ of dietary tryptophan is catabolized via IDO in the form of kynurenine degradation products (marked by red arrows). Additional enzymatic metabolites in the pathway stimulate pathologies due to insufficient or excessive immune suppression. KAT, kynurenine aminotransferase; MAO, monoamine oxidase; HAO, 3-hydroxyanthranilic acid oxidase; IDO, indoleamine 2, 3-dioxygenase; Th, T helper cells.

blockade of mTOR by IDO was reported to trigger autophagy in anergized $\mathrm{T}$ cells, whereas the opposite effect was observed in tryptophan restoration, which relieved mTOR blockade (18). The observation of blockade of mTOR by IDO suggests it may act as a pivotal regulator, however direct effects of IDO on the mTOR pathway have not yet been fully elucidated, and further research is required to verify the specific mechanism.

Effector pathways: Tryptophan catabolites as ligands for AhR. In addition to GCN2 kinase, it has previously been demonstrated that the breakdown products of tryptophan catabolism are important crucial mediators in the IDO pathway. It was revealed that high IDO expression and tryptophan metabolites (3-hydroxykynurenine and 3-hydroxyanthranilic acid) in transgenic DCs, irreversibly inhibit allogeneic T-cell proliferation. Conversely, increased kynurenine/tryptophan ratios in plasma $\mathrm{IDO}^{+} \mathrm{DC}$ may promote the production of Tregs and induce the effector $\mathrm{T}$ cells in the state of anergy or apoptosis. Kynurenine catabolites exert a cytotoxic function on $\mathrm{CD}^{+}$ cells, however the molecular mechanism remains to be fully elucidated, and potentially involves in inhibition of $\mathrm{T}$ cell costimulatory signaling via 3 '-phosphoinositide-dependent kinase 1 (an essential mediator of CD28-induced NF- $\mathrm{BB}$ activation) pathway and activation of signaling via AhR (19).

$\mathrm{AhR}$ is a transcription factor that is activated by dioxin-like kynurenine-related ligands. AhR has previously been implicated in the inflammatory and immune regulations that IDO is involved in. The immunological effects of AhR activation on $\mathrm{T}$ cell subsets appear to be immunosuppressive, including arrest of $\mathrm{T}$ cell activation, induction of differentiation of Foxp $3^{+}$Treg cells, alterations in the functional immunogenicity of DCs and suppression of anti-tumor immune responses (20). AhR signaling in DCs is additionally required to induce the expression of functional IDO, indicating occurrence of crosstalk between the two pathways. Kynurenine regulates an effector signaling pathway from IDO in activating AhR, however additionally regulates tryptophan catabolizing enzyme, tryptophan 2,3-dioxygenase, which has the capacity to inhibit anti-tumor immune responses and promote tumor cell survival (21).
Metabolites of tryptophan are directly toxic to $\mathrm{CD}^{+} \mathrm{T}$ cells and CD $4{ }^{+}$Th1 cells, however not to Th2 cells, therefore enhanced IDO activity appears to redirect $\mathrm{T}$ helper cell polarization toward a Th2 phenotype. IDO activity may be partially counteracted by two negative feedback loops, including kynurenine increasing IL-6 expression through AhR and eIF-2 $\alpha$, leading to incremental B-lymphocyte induced maturation protein 1 levels, which impede the INDO promoter region (22). This allows for fine tuning of IDO activity to maintain a balance between immune activation and suppression, as necessary. Therapeutically, administration of kynurenine compounds may protect transplanted tissues from an inflammatory reaction response and promote immune tolerance.

Effector pathways: A signaling protein in $N F-\kappa B$ pathway. It was indicated that IDO may function as a signaling protein responsible for the self-amplification and maintenance of a stably regulatory phenotype in plasmacytoid dendritic cells (pDCs) rather than a catalyst. For pDCs treated with transforming TGF- $\beta$ in mice, this signaling function occurs via recruitment and activation of Src homology region 2 domain-containing phosphatase proteins bonding to immunoreceptor tyrosine-based inhibitory motifs in the Fyn-dependent phosphorylation of IDO molecule. IDO then initiates a circuit of downstream signaling effectors, including the noncanonical $\mathrm{NF}-\kappa \mathrm{B}$ pathway, that result in sustained tumor growth factor (TGF)- $\beta$ production, induction of type I interferons and the regulatory $\mathrm{pDC}$ phenotype, ultimately inducing long-lasting IDO expression and autocrine TGF- $\beta$ secretion in a positive feedback loop (23). Furthermore, noncanonical NF- $\kappa \mathrm{B}$ signaling downregulates proinflammatory cytokine production in DCs, and selective activation of the noncanonical NF- $\kappa \mathrm{B}$ pathway gives rise to noninflammatory DCs that suppress $\mathrm{T}$ cell activation and promote the development of $\mathrm{T}$ cells with regulatory properties (24). Accordingly, noncanonical $\mathrm{NF}-\kappa \mathrm{B}$ signaling in DCs is required for IDO induction and immune regulation.

Regulatory effect of IDO on T cells and Tregs. IDO pathways have important effects on $\mathrm{T}$ cells in response to antigenic 


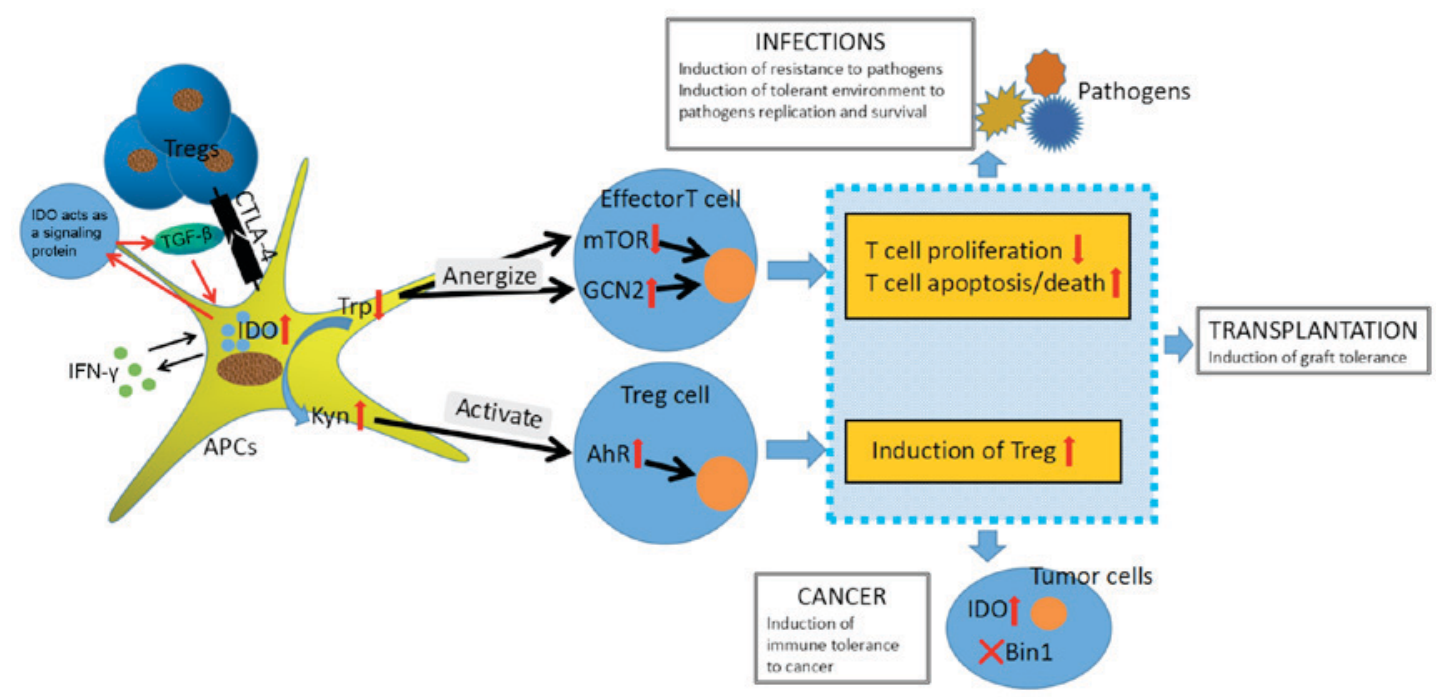

Figure 2. IDO pathway control of T cell and Treg responses. IDO is expressed on professional APCs and tumor cells, and is critical in immune regulation of cancers, transplantation and infections by catalyzing oxidative catabolism of the essential amino acid tryptophan, along the kynurenine pathway. IDO modifies immune response in three pathways: By depleting tryptophan in the local microenvironment, IDO results in metabolic stress sensed by GCN2 kinase and mTOR, which eventually promotes anergy in responding T cells and directs the conversion of Foxp $3^{+} \mathrm{T}$ cell; by producing tryptophan catabolism that binds to a natural ligand for AhR, IDO similarly achieves immunosuppressive effects on the immunogenicity of dendritic cell reduction and the Foxp $3^{+}$Tregs conversion; by inducing regulatory $\mathrm{T}$ cell function, IDO as a signaling protein shapes the immunological microenviroment in vivo. IDO, indoleamine 2, 3-dioxygenase; mTOR, mammalian target of Rapamycin; GCN2, general control nondepressible-2; APC, antigen presenting cell; AhR, aryl hydrocarbon receptor; CTLA4, cytotoxic T-lymphocyte antigen 4; Foxp3 $3^{+}$, Forkhead Box P3; Treg, T regulatory cells; TGF- $\beta$, tumor growth factor- $\beta$; IFN- $\gamma$, interferon- $\beta$; Trp, tryptophan; Kyn, kynurenine; BIN-1, bridging integrator 1.

stimulation (Fig. 2). T cells activated by DCs expressing IDO recognize the antigen and enter into cell cycle, however IDO-induced activation of GCN2 blocks subsequent cell cycle progression, leading to the inhibition of Th17 differentiation and the increase of $\mathrm{T}$ cell apoptosis. The IDO activity fails to regulate $\mathrm{T}$ cells in the case of GCN2 dependent amino acid stress response pathways defected $(13,15)$. Additionally, the IDO-mediated redox in DCs may affect T cell sensitivity. In a model of pulmonary aspergillosis in mice, a superoxide-dependent step in tryptophan metabolism along the kynurenine pathway is inhibited, leading to unrestrained T-cell reactivity, dominant production of IL-17, and defective regulatory T-cell activity (25). However, whether T cells are regulated by IDO activity solely in DCs that present antigens directly to T cells, or whether T cells activated by DCs not expressing IDO would be affected by local IDO activity in bystander DCs, remains to be clarified.

The majority of immune responses are naturally blocked by increasing $\mathrm{CD} 4{ }^{+} \mathrm{CD} 25^{+} \mathrm{Foxp} 3^{+}$Tregs. The IDO pathway is conducive to regulation of Treg lineage commitment and function. In vitro, tryptophan depletion (sensed by GCN2) acts synergistically with kynurenine metabolites to redirect $\mathrm{CD}^{+}$ T cells to differentiate into Foxp $3^{+}$Treg cells. In vivo, inhibition or knockout of IDO genes prevents the antigen-specific Tregs in response to pathogen challenge and decreases the ratio of $\mathrm{T}$ regulatory/T effector cells (26). The $\mathrm{B} 7$ receptors on DCs expressing IDO bind to cytotoxic T-lymphocyte antigen 4 (CTLA4) on Tregs, resulting in their proliferation and antigen-specific anergy $(5,11)$.Under conditions of IDO ablation, resting Tregs convert uniformly into a phenotype resembling proinflammatory Th17 cells. The reprogrammed Treg cells following IDO-blocking have been characterized as analogous to that of 'polyfunctional' T-helper cells co-expressing IL-17,
IL-2 and tumor necrosis factor- $\alpha$ (27). Therefore, IDO is important in the differentiation of Foxp3 ${ }^{+}$Tregs to Th17-like effector cells. IDO stimulates Treg cell bystander suppressor activity and simultaneously inhibits the IL-6 secretion, which is required for the conversion of Tregs into Th17-like effector cells. Notably, human monocyte-derived DCs upregulate IDO expression induced by proinflammatory cytokines, expanding the population of allogenetic autologous Tregs. These Tregs suppress autologous and allogeneic proliferation of T-cells and repress the generation of antigen-specific CTL (28).

Therefore, IDO pathways and IDO-mediated tryptophan degradation, regulate the balance between effector $\mathrm{T}$ cells and Tregs in favor of Tregs. This transition may control excess inflammation and prevent immune-mediated pathology. However, whether IDO represents a predominant mechanism or operates in a synergistic manner in combination with other tolerogenic effects, requires further investigation.

\section{Role of IDO in immune suppression}

The role of IDO in cancer. IDO is widely overexpressed in tumor cells and acts at multifarious levels to establish a more hospitable environment for tumor progression. It has previously been demonstrated that increased expression of IDO is predominantly associated with poor prognosis in a variety of cancer types, including ovarian, endometrial, colorectal and cervical cancers (29-30). Studies using murine models have indicated that IDO expression is not always present in tumor cells, however may be located in tumor-draining lymph nodes or in surrounding stroma near the tumor margins (31). IDO activation in either tumor cells or nodal regulatory DCs appears to be adequate to facilitate tumor immune escape. There are two potential sites for the immunosuppressive action of IDO in 
tumor-bearing hosts: Firstly, IDO expressed by the tumor cells enables creation of a localized immunosuppressive milieu in the tumor, either by inhibiting effector function and proliferation of $\mathrm{T}$ cells in the tumor, or by utilizing toxic metabolites of tryptophan to directly kill infiltrating T cells. Alternatively, host DCs expressing IDO are able to identify tumor-derived antigens and transfer them to tumor-draining lymph nodes where they would present the antigen to naive $T$ cells for induction of T-cell deletion, a failure of clonal expansion, or perhaps even the biasing of various cells toward a regulatory phenotype. DCs respond to low tryptophan by increasing expression of the inhibitory receptors Ig-like transcript 3 (ILT3), ILT4 and TGF- $\beta 1$, which renders them immunosuppressive APCs (32). Due to the potent immunological functions of IDO, upregulation of IDO by host APCs or tumor cells may be critical for the induction of the tumor-tolerant environment.

Mouse genetic research has demonstrated that IDO overexpression may be regulated by inactivation of bridging integrator (Bin)1 (a tumor suppressor gene), which appears to prevent cancer development to a significant extent by limiting immune escape (6). However, Bin1 expression in human tumors is extinguished by aberrant RNA splicing patterns and altered gene methylation patterns. Furthermore, it has been indicated that inactivated Bin1 generates cancer cell-intrinsic benefits for cell proliferation and survival. In an in vitro study, transformation of Bin1-null and Bin1-expressing primary mouse embryo keratinocytes with oncogenes, produced cell lines which then were grafted into syngeneic animals. The Bin1-null cells appeared to form larger tumors, whereas only indolent nodules were seen in the Bin1-expressing cell group. Overall, overexpression of IDO accompanied by Bin1 inactivation or loss promotes tumorigenesis by enabling immune escape (33).

The role of IDO in transplantation. Successful and sustained allograft tolerance is based on effective control of the potential immune reaction. Current treatments employ a general immunosuppressant, which results in patient susceptibility to pathogenic infection and potentially severe adverse effects. Mice with IDO knockout experience acute rejection injury of transplanted major histocompatibility complex mismatched grafts, whereas long-term survival occurs in wild-type mice with high-level tryptophan catabolism (34). Further experiments of liver transplantation have demonstrated that IDO expression of Kupffer cells demonstrates a time-dependent increase in the tolerance group, and the number of IDO-positive cells are closely associated with the severity of acute reaction (35). A further study demonstrated that $\mathrm{IDO}^{+} \mathrm{DC}$ transfusion prolongs the survival of recipients in small bowel transplantation models, and more efficient results may be obtained with 3-hydroxyanthranilic acid treatment (36). IDO induction of DC is dependent on transcription factor Foxp3. Surface CTLA-4 expressed by Treg cells binds B7 molecules on DCs to induce IDO expression and promote a DC regulatory phenotype, and this phenomenon from CTLA-4 to B7 is likely modulated by induced Foxp $3^{+}$Treg cells. In addition, IDO may activate Treg cells via the aforementioned AhR and GCN2 pathways. Therefore, there is latent for a mutually reinforcing loop, which maintains a lasting transplant tolerance microenvironment. Additionally, in a study of rat lung allografts, functionally inhibiting cytotoxic $\mathrm{CD} 8^{+} \mathrm{T}$ cells were demonstrated to be critical in the mechanism of immune modulation of IDO, which reduces infiltrating $\mathrm{CD} 8^{+} \mathrm{T}$ cells and impairs cytotoxic function on perforin and granzyme A/B secretion (37).

The role of IDO in infectious diseases. In infectious disease states, IDO exerts pleiotropic effects, acting as a suppressor of intracellular pathogen replication and as an immune regulator. IFN $-\beta$ and IFN $-\gamma$ induced tryptophan degradation leads to enhanced IDO activity against pathogens including cytomegalovirus, Herpes simplex virus type 2, Chlamydia psittaci strains and Leishmania donovani (5). A clinical experiment demonstrated that IDO expression increases in chronically infected hepatitis $\mathrm{C}$ patients and acute hepatitis B patients, however not in those from recovered patients or patients with hepatic flare $(38,39)$, suggesting that IDO may be an indicator of subsequent immune responses operative during the early phase of infection. Conversely, pathogens are capable of highjacking the immunosuppressive effects of IDO and using them to facilitate their own life cycle. Leishmania parasites circumvent immune clearance via promoting the induction of IDO among host DCs. The immunocompromised response on IDO induction enables a triumphant localization of Leishmania (40). IDO may be crucial in forming pathogen-induced lung inflammation in the influenza infection, and predisposes the lung to secondary bacterial infection. Inhibition of IDO activity contributes to the activation of the heterosubtypic memory $\mathrm{T}$ cell response for cross-protective immunity against the influenza virus (41).

In particular, the human immune deficiency virus (HIV) may induce IDO synthesis to escape the direct killing mechanism of $\mathrm{CD}^{+}$cytotoxic lymphocyte recognition. The induction of IDO in APCs via the N-terminal domain of HIV-1 transactivator regulatory protein (Tat) is engendered through an intracellular signaling cascade reaction including Janus activated kinases (Jak) I, phosphatidyl inositol 3-kinase (PI3K) or CTLA-4-B7 interaction, which consequently results in a breakdown of tryptophan to kynurenine and a suppression of T-cell proliferation. IFN- $\gamma$ signaling resulting in IDO expression may be blocked by JAKs and PI3K inhibitors, however this does not occur with Tat-induced IDO expression, suggesting further investigation is necessary in order to elucidate the novel mechanism underlying IDO induction from Tat proteins in HIV infection (42). In HIV patients, it has been observed that elevated IDO enzymatic activity in APCs is negatively associated with Th22:Treg and Th17:Treg ratios in the anti-retroviral therapy-naive group, suggesting that imbalance of the ratio of Th22/Th17 to Tregs may contribute to widespread immune dysfunction in HIV-1 infection (43).

\section{Strategies to target IDO}

Currently, a broad range of candidate compounds have been developed as IDO-inhibitors for clinical application. Of the IDO inhibitors, 1-methyl-DL-tryptophan (1-MT) is the most extensively studied. There are two available stereoisomers of 1-MT; D and L isomers. L-1MT is advantageous in suppressing the enzymatic activity of IDO (tryptophan degradation into kynurenine) in cell lines. The D-1MT stereoisomer induces T-cell proliferation in allogeneic mixed lymphocyte reactions. IDO-mediated products of tryptophan repress the 
immunoregulatory kinases mTOR and PKC- $\theta$, which may be relieved by D-1MT. D-1MT acts as a potential tryptophan mimetic in mTOR regulation by restoration of mTOR pathway. Conversely, the immunostimulatory effect of L-1MT is restricted by activation of the AhR pathway in response to production of N-methyl-kynurenine (44). Overall, D-1MT exhibits a broader range of benefits and is therefore clinically applied to a greater extent, compared with L-1MT.

Additionally, IDO blockade enhances the effectiveness of chemotherapy. Mice administered with IDO-inhibitor plus disparate chemotherapeutic agents, including cyclophosphamide, doxorubicin, or cisplatin, congruously demonstrate smaller tumors compared with those treated with hemotherapeutic agents alone (45). However, it remains unclear whether this effect may occur as a result of various roles of IDO in restoring Treg-mediated suppression following chemotherapy. Regarding in-situ modification of Tregs, therapeutic anti-tumor vaccinations have improved immune responses in mice with B16 melanoma tumors (46), inducing extensive conversion of Tregs into polyfunctional 'reprogrammed' IL-17 expressing Th17 cells. Other immune modulators, including anti-CTLA-4 monoclonal antibodies, may ultimately be associated with IDO inhibitors in therapeutic application.

\section{Conclusions}

In conclusion, IDO is a physiological host mechanism for immunological tolerance in various settings. IDO functions at the level of metabolic regulation and effects the activation or inhibition of immunity and cellular metabolism via controlling pathways including GCN2, mTOR and AhR. The pivotal role of IDO in immune inhibition is dependent on the depletion of cellular tryptophan levels and the generation of kynurenines that result in T effector cell anergy and induce the proliferation of Tregs. Therefore, regulation of IDO biosynthesis or activity in the immune system exhibits immunological implication in various biological processes, including cancer, transplantation and infection. The targeting of IDO is currently applied in clinics as a therapeutic strategy, however, further investigations are required in order to fully elucidate the mechanisms of the various pathways affected by IDO activation.

\section{Competing interests}

The authors declare that they have no competing interests.

\section{References}

1. Munn DH: Indoleamine 2,3-dioxygenase, Tregs and cancer. Curr Med Chem 18: 2240-2246, 2011.

2. Gerriets VA and Rathmell JC: Metabolic pathways in T cell fate and function. Trends Immunol 33: 168-73, 2012.

3. Munn DH, Zhou M, Attwood JT, Bondarev I, Conway SJ, Marshall B, Brown C and Mellor AL: Prevention of allogeneic fetal rejection by tryptophan catabolism. Science 281: 1191-1193, 1998.

4. Ciorba MA, Bettonville EE, McDonald KG, Metz R, Prendergast GC, Newberry RD and Stenson WF: Induction of IDO-1 by immunostimulatory DNA limits severity of experimental colitis. J Immunol 184: 3907-3916, 2010.

5. Mbongue JC, Nicholas DA, Torrez TW, Kim NS, Firek AF and Langridge WH: The Role of indoleamine 2, 3-dioxygenase in immune suppression and autoimmunity. Vaccines (Basel) 3: $703-729,2015$
6. Prendergast GC, Smith C, Thomas S, Mandik-Nayak L, Laury-Kleintop L, Metz R and Muller AJ: Indoleamine 2,3-dioxygenase pathways of pathogenic inflammation and immune escape in cancer. Cancer Immunol Immunother 63: 721-735, 2014.

7. Trabanelli S, Ocadlikova D, Evangelisti C, Parisi S and Curti A: Induction or regulatory $\mathrm{T}$ Cells by dendritic cells through indoleamine 2,3-dioxygenase: A potent mechanism of acquired peripheral tolerance. Curr Med Chem 18: 2234-2239, 2011.

8. Huang L, Baban B, Johnson BA and Mellor AL: Dendritic cells, indoleamine 2,3 dioxygenase and acquired immune privilege. Int Rev Immunol 29: 133-155, 2010.

9. Ball HJ, Sanchez-Perez A, Weiser S, Austin CJ, Astelbauer F, Miu J, McQuillan JA, Stocker R, Jermiin LS and Hunt NH: Characterization of an indoleamine 2,3-dioxygenase-like protein found in humans and mice. Gene 396: 203-213, 2007.

10. Poormasjedi-Meibod MS, Jalili RB, Hosseini-Tabatabaei A, Hartwell R and Ghahary A: Immuno-Regulatory Function of Indoleamine 2,3 Dioxygenase through Modulation of Innate Immune Responses. PLoS One 8: e71044, 2013.

11. Munn DH and Mellor AL: Indoleamine 2,3 dioxygenase and metabolic control of immune responses. Trends Immunol 34: 137-143, 2013.

12. Nguyen NT, Kimura A, Nakahama T, Chinen I, Masuda K, Nohara K, Fujii-Kuriyama Y and Kishimoto T: Aryl hydrocarbon receptor negatively regulates dendritic cell immunogenicity via a kynurenine-dependent mechanism. Proc Natl Acad Sci USA 107: 19961-19966, 2010.

13. Sundrud MS, Koralov SB, Feuerer M, Calado DP, Kozhaya AE, Rhule-Smith A, Lefebvre RE, Unutmaz D, Mazitschek R, Waldner $\mathrm{H}$, et al: Halofuginone inhibits $\mathrm{T}_{\mathrm{H}} 17$ cell differentiation by activating the amino acid starvation response. Science 324 : 1334-1338, 2009

14. Baban B, Chandler PR, Johnson BA, Huang L, Li M, Sharpe ML, Francisco LM, Sharpe AH, Blazar BR, Munn DH, et al: Physiologic control of IDO competence in splenic dendritic cells. J Immunol 187: 2329-2335, 2011.

15. Smith C, Chang MY, Parker KH, Beury DW, DuHadaway JB, Flick HE, Boulden J, Sutanto-Ward E, Soler AP, Laury-Kleintop LD, et al: IDO Is a nodal pathogenic driver of lung cancer and metastasis development. Cancer Discov 2: 722-735, 2012.

16. Chaudhary K, Shinde R, Liu H, Gnana-Prakasam JP, Veeranan-Karmegam R, Huang L, Ravishankar B, Bradley J, Kvirkvelia N, McMenamin M, et al: Amino acid metabolism inhibits antibody-driven kidney injury by inducing autophagy. J Immunol 194: 5713-5724, 2015.

17. Chuang HC, Lan JL, Chen DY, Yang CY, Chen YM, Li JP, Huang CY, Liu PE, Wang $X$ and Tan TH: The kinase GLK controls autoimmunity and NF- $\kappa \mathrm{B}$ signaling by activating the kinase PKC- $\theta$ in T cells. Nat Immunol 12: 1113-1138, 2011.

18. Metz R, Rust S, Duhadaway JB, Mautino MR, Munn DH, Vahanian NN, Link CJ and Prendergast GC: IDO inhibits a tryptophan sufficiency signal that stimulates mTOR: A novel IDO effector pathway targeted by D-1-methyl-tryptophan. Oncoimmunology 1:1460-1468, 2012.

19. Zaher SS, Germain C, Fu H, Larkin DF and George AJ: 3-Hydroxykynurenine suppresses CD4+ T-cell proliferation, induces T-regulatory-cell development, and prolongs corneal allograft survival. Investig. Invest Ophthalmol Vis Sci 52: 2640-2648, 2011.

20. Hao K, Zhou Q, Chen W, Jia W, Zheng J, Kang J, Wang K and Duan T: Possible role of the 'IDO-AhR axis' in maternal-foetal tolerance. Cell Biol Int 37: 105-108, 2013.

21. Pilotte L, Larrieu P, Stroobant V, Colau D, Dolusic E, Frédérick R, De Plaen E, Uyttenhove C, Wouters J, Masereel B and Van den Eynde BJ: Reversal of tumoral immune resistance by inhibition of tryptophan 2,3-dioxygenase. Proc Natl Acad Sci USA 109: 2497-2502, 2012.

22. DiNatale BC, Murray IA, Schroeder JC, Flaveny CA, Lahoti TS, Laurenzana EM, Omiecinski CJ and Perdew GH: Kynurenic acid is a potent endogenous aryl hydrocarbon receptor ligand that synergistically induces interleukin- 6 in the presence of inflammatory signaling. Toxicol Sci 115: 89-97, 2010.

23. Pallotta MT, Orabona C, Volpi C, Vacca C, Belladonna ML, Bianchi R, Servillo G, Brunacci C, Calvitti M, Bicciato S, et al: Indoleamine 2,3-dioxygenase is a signaling protein in long-term tolerance by dendritic cells. Nat Immunol 12: 870-878, 2011. 
24. Tas SW, Vervoordeldonk MJ, Hajji N, Schuitemaker JH, van der Sluijs KF, May MJ, Ghosh S, Kapsenberg ML, Tak PP and de Jong EC: Noncanonical NF-kappaB signaling in dendritic cells is required for indoleamine 2,3-dioxygenase (IDO) induction and immune regulation. Blood 110: 1540-1549, 2007.

25. Romani L, Fallarino F, De Luca A, Montagnoli C, D'Angelo C, Zelante T, Vacca C, Bistoni F, Fioretti MC, Grohmann U, et al: Defective tryptophan catabolism underlies inflammation in mouse chronic granulomatous disease. Nature 451: 211-215, 2008.

26. Matteoli G, Mazzini E, Iliev ID, Mileti E, Fallarino F, Puccetti P, Chieppa M and Rescigno M: Gut CD103+ dendritic cells express indoleamine 2,3-dioxygenase which influences $\mathrm{T}$ regulatory/T effector cell balance and oral tolerance induction. Gut 59: 595-604, 2010

27. Sharma MD, Hou DY, Liu Y, Koni PA, Metz R, Chandler P, Mellor AL, He Y and Munn DH: Indoleamine 2,3-dioxygenase controls conversion of Foxp3? Tregs to TH17-like cells in tumor-draining lymph nodes. Blood 113: 6102-6111, 2009.

28. Chung DJ, Rossi M, Romano E, Ghith J, Yuan J, Munn DH and Young JW: Indoleamine 2,3-dioxygenase-expressing mature human monocyte-derived dendritic cells expand potent autologous regulatory T cells. Blood 114: 555-563, 2009.

29. Thaker AI, Rao MS, Bishnupuri KS, Kerr TA, Foster L, Marinshaw JM, Newberry RD, Stenson WF and Ciorba MA IDO1 metabolites activate beta-catenin signaling to promote cancer cell proliferation and colon tumorigenesis in mice. Gastroenterology 145: 416-425.e1-4, 2013.

30. Ferns DM, Kema IP, Buist MR, Nijman HW, Kenter GG and Jordanova ES: Indoleamine-2,3-dioxygenase (IDO) metabolic activity is detrimental for cervical cancer patient survival. Oncoimmunology 4: e981457, 2015.

31. Johnson TS, Munn DH and Maria BL: Modulation of tumor tolerance in primary central nervous system malignancies. Clin Dev Immunol 2012: 937253, 2012.

32. Munn DH and Mellor AL: IDO and tolerance to tumors. Trends Mol Med 10: 15-18, 2004

33. Palucka K, Banchereau J and Mellman I: Designing vaccines based on biology of human dendritic cell subsets. Immunity 33 464-478, 2010.

34. Brandacher G, Cakar F, Winkler C, Schneeberger S, Obrist P, Bösmüller C, Werner-Felmayer G, Werner ER, Bonatti H, Margreiter R and Fuchs D: Non-invasive monitoring of kidney allograft rejection through IDO metabolism evaluation. Kidney Int 71: 60-67, 2007.

35. Sun X, Gong ZJ, Wang ZW, Li T, Zhang JY, Sun HC, Liu S, Huang L, Huang $\mathrm{C}$ and Peng ZH: IDO-Competent-DCs induced by IFN- $\gamma$ attenuate acute rejection in rat liver transplantation. J Clin Immunol 32: 837-847, 2012.
36. Xie FT, Cao JS, Zhao J, Yu Y, Qi F and Dai XC: IDO expressing dendritic cells suppress allograft rejection of small bowel transplantation in mice by expansion of Foxp3+ regulatory T cells. Transpl Immunol 33: 69-77, 2015.

37. Liu WL, Lin YH, Xiao H, Xing S, Chen H, Chi PD and Zhang G: Epstein-Barr virus infection induces indoleamine 2, 3-dioxygenase expression in human monocyte-derived macrophages through p38/mitogen-activated protein kinase and NF- $\kappa \mathrm{B}$ pathways: Impairment in T cell functions. J Virol 88: 6660-6671, 2014.

38. Schulz S, Landi A, Garg R, Wilson JA and van Drunen Littel-van den Hurk S: Indolamine 2,3-dioxygenase expression by monocytes and dendritic cell populations in hepatitis $\mathrm{C}$ patients. Clin Exp Immunol 180: 484-498, 2015.

39. Yoshio S, Sugiyama M, Shoji H, Mano Y, Mita E, Okamoto T, Matsuura Y, Okuno A, Takikawa O, Mizokami M and Kanto T: Indoleamine-2,3-dioxygenase as an effector and an indicator of protective immune responses in patients with acute hepatitis B. Hepatology 63: 83-94, 2016.

40. Donovan MJ, Tripathi V, Favila MA, Geraci NS, Lange MC, Ballhorn W and McDowell MA: Indoleamine 2,3-dioxygenase (IDO) induced by Leishmania infection of human dendritic cells. Parasite Immunol 34: 464-472, 2012.

41. Sage LK, Fox JM, Mellor AL, Tompkins SM and Tripp RA: Indoleamine 2,3-dioxygenase (IDO) activity during the primary immune response to influenza infection modifies the memory $\mathrm{T}$ Cell response to influenza challenge. Viral Immunol 27: 112-123, 2014.

42. Planès R and Bahraoui E: HIV-1 Tat protein induces the production of IDO in human monocyte derived-dendritic cells through a direct mechanism: Effect on T cells proliferation. PLoS One 8: e74551, 2013

43. Page EE, Greathead L, Metcalf R, Clark SA, Hart M, Fuchs D, Pantelidis P, Gotch F, Pozniak A, Nelson M, et al: Loss of Th22 cells is associated with increased immune activation and IDO-1 activity in HIV-1 infection. J Acquir Immune Defic Syndr 67: 227-235, 2014.

44. Moon YW, Hajjar J, Hwu P and Naing A: Targeting the indoleamine 2,3-dioxygenase pathway in cancer. J Immunother Cancer 3: 51, 2015.

45. Andersen $\mathrm{MH}$ : The specific targeting of immune regulation: T-cell responses against Indoleamine 2,3-dioxygenase. Cancer Immunol Immunother 6: 1289-1297, 2012.

46. Sharma MD, Hou DY, Baban B, Koni PA, He Y, Chandler PR, Blazar BR, Mellor AL and Munn DH: Reprogrammed foxp3(+) regulatory $\mathrm{T}$ cells provide essential help to support cross-presentation and CD8(+) T cell priming in naive mice. Immunity 33 : 942-954, 2010 